\title{
Contribuições Teóricas sobre o Estudo de Redes de Conhecimento Interorganizacionais
}

Theoretical Contributions on Inter-Organizational Knowledge Networks Studies

Rodrigo Müller ${ }^{1}$ e Faimara do Rocio Strauhs ${ }^{1}$

${ }^{1}$ Universidade Tecnológica Federal do Paraná, UTFPR 


\title{
Resumo
}

As redes de conhecimento $(\mathrm{RC})$ interorganizacionais podem ser vistas como espaços de interação entre os mais variados grupos e/ou segmentos da sociedade, visando à cooperação, ao compartilhamento de informações, aos recursos, aos conhecimentos e às experiências. A existência das RC nos mais variados ambientes pode auxiliar organizações e indivíduos nos processos de compartilhamento e de criação de novos conhecimentos. Nesse contexto, esta pesquisa busca identificar as características das redes de conhecimento e investigar como ocorre a criação do conhecimento dessas redes. A pesquisa desenvolve-se sob a metodologia teórico-exploratória, buscando identificar na literatura específica, por meio de uma pesquisa bibliométrica, os principais constructos da área, bem como as características dos ambientes de cooperação. Após a coleta de dados e a análise prévia dos materiais encontrados, verificou-se que o estudo das RC é recente e possui, ainda, poucos trabalhos. No cenário brasileiro, a pequena quantidade de trabalhos encontrados demonstra que o tema é incipiente, carecendo de estudos e pesquisas direcionadas ao cenário nacional. Já no campo internacional, vê-se uma maior quantidade de trabalhos publicados, no entanto com poucas abordagens empíricas. Após estas análises é possível perceber que as RC são dinâmicas e dependem da criação de laços de confiança entre as organizações participantes. É possível ainda identificar que a maioria das redes atuais se configura a partir da identificação de necessidades semelhantes entre empresas diferentes, o que indica uma posição de abertura dessas organizações para o trabalho conjunto e o compartilhamento de recursos, informações e conhecimentos.

Palavras-chave: redes de conhecimento interorganizacionais, cooperação interorganizacional, compartilhamento do conhecimento

\begin{abstract}
Inter-organizational knowledge networks (KN) may be seen as spaces for interaction among the most varied groups and/ or segments of society, aiming at cooperation, sharing information, resources, knowledge and experiences. The existence of $K N$ in the most varied environments can help organizations and individuals in the processes of sharing and creating new knowledge. In this context, this research aims to identify the characteristics of knowledge networks and investigate how the creation of knowledge in these networks occurs. The research is developed under the theoretical-exploratory methodology, seeking to identify in the specific literature, by a bibliometric research, the main constructs of the area, as well to identify the characteristics of the cooperation environments. After data collection and prior analysis of the materials, it was found that the study of $K N$ is recent and thus has few studies. In the Brazilian scenario, the small number of studies found shows that the topic is still incipient, lacking studies and research focused on the national scenario. In the international field, however, there is a greater number of published works, but few based on empirical approaches. After these analysis it is possible to conclude that the $K N$ are dynamic and depend on the creation of bonds of trust between the participating organizations. It is also possible to identify that most of current networks are configured based on the identification of similar needs between different companies, which indicates a position of openness of these organizations to joint work and sharing resources, information and knowledge.
\end{abstract}


O complexo cenário econômico e social de grande parte das organizações contemporâneas tem exigido posturas que consigam responder às demandas de informações e de conhecimentos, tanto internas como externas a essas organizações. Em se tratando do conhecimento, tal cenário se torna ainda mais exigente na medida em que produtos e serviços se baseiam cada vez mais na utilização e agregação de conhecimentos diversos para sua elaboração.

Considerando que o conhecimento é um elemento que pertence às pessoas, frequentemente tácito e de difícil articulação (Polanyi, 1958), surge o desafio das empresas de lidar com esse recurso de forma a aplicá-lo em seus produtos, serviços e processos organizacionais. No entanto, nem sempre os conhecimentos e os recursos necessários para a realização das atividades das organizações estão disponíveis internamente. Assim, é preciso buscar além das fronteiras institucionais e, em alguns casos, nas ações de cooperação com outras organizações os recursos físicos, estruturais, financeiros, de informação e de conhecimento necessários.

Nesse contexto encontram-se as redes de cooperação interorganizacionais, trazendo a possibilidade de compartilhamento de recursos e de desenvolvimento de conhecimentos por meio de ações conjuntas. Um exemplo dessas redes são as redes de conhecimento (RC), que têm por finalidade o compartilhamento e a criação de conhecimentos entre os membros da rede, de forma que todos tenham acesso aos benefícios advindos das ações cooperativas e da efetividade do grupo.

Partindo desses elementos, são objetivos do presente ensaio identificar as características do ambiente de cooperação interorganizacional e das redes de conhecimento e investigar como ocorre a criação do conhecimento organizacional dentro das redes interorganizacionais.

A metodologia utilizada é a investigação teórico-exploratória, contando com uma pesquisa bibliográfica subsidiada pela pesquisa bibliométrica sobre os temas em questão. Os resultados encontrados na pesquisa bibliométrica são utilizados para compor um panorama teórico dos estudos e pesquisas sobre as redes de conhecimento.

Nesse sentido, este trabalho está dividido em cinco seções, iniciando-se por esta introdução, que apresenta os direcionamentos gerais do trabalho. Em seguida, na seção 2 são apresentados os conceitos teóricos utilizados e os resultados da pesquisa bibliográfica. $\mathrm{O}$ item 3 apresenta a metodologia adotada para a realização da pesquisa bibliométrica e das discussões. Já a seção 4 traz as discussões e resultados advindos da revisão da literatura identificada na pesquisa bibliométrica. Por fim, na seção 5 , apresentam-se as principais conclusões deste estudo apontando características e elementos constituintes das 'redes de conhecimento', seguidas da bibliografia consultada.

\section{Referencial Teórico}

Nesta seção, são abordados os temas de redes de cooperação e de redes de conhecimento, buscandose evidenciar as características desses tipos de redes e os elementos básicos entre elas. Por meio da revisão da literatura, pode-se perceber a relação entre os temas e as complementaridades entre eles, conforme apresentado nas próximas seções. 


\section{Redes de Cooperação}

Para Balestrin e Verschoore (2009), há tempos a colaboração entre empresas acontece. No entanto, "mais recentemente, a expansão tecnológica global ampliou a capacidade conectiva das organizações e as possibilidades de ações conjuntas" (p. 24). Para os autores, as aglomerações na forma de redes de cooperação reúnem empreendimentos com objetivos comuns e, em alguns casos, inter-relacionados, com o intuito de desenvolver ganhos mútuos, sem que, no entanto, cada participante perca sua autonomia de gestão.

Nesse sentido, as organizações contemporâneas buscam nas ações de cooperação a complementaridade que necessitam não apenas para as ações de inovação, mas para outros processos e demandas, como o próprio aprendizado organizacional, que pode, no longo prazo, resultar em inovações diversas.

Já DeBresson e Amesse (1991) apontam que por meio das redes de colaboração é possível combinar elementos, conhecimentos e recursos diferentes que podem dar origem a inovações variadas dentro das empresas participantes dos grupos, ou redes de cooperação. No entanto, os autores salientam que, para serem efetivas, as redes de cooperação devem alcançar uma coesão em suas ações, de forma que todos os participantes sejam beneficiados.

No entanto, uma das principais características, e também um dos principais condicionantes, para a atuação em rede é a interação. Para que sejam efetivas, as redes devem manter relações interativas entre seus participantes, onde dados, informações, conhecimentos e outros recursos possam ser compartilhados mutuamente (Johnson, 2011). Essa dinâmica, entretanto, é complexa, assim como o ambiente empresarial contemporâneo. Por outro lado, situações complexas demandam abordagens complexas e sistêmicas, de forma que a atuação em rede por parte das empresas se torna uma alternativa e uma forma de resposta ao contexto socioeconômico atual.

Conforme aponta Johnson (2011), em ambientes complexos as organizações desenvolvem capacidades de lidar com a incerteza e com as variadas demandas ambientais de forma mais rápida, uma vez que essas demandas podem interferir diretamente na sobrevivência organizacional.

No cenário das organizações contemporâneas, tal complexidade aumenta na medida em que muitas empresas mantêm vínculos que vão além das relações comerciais, ou das relações tradicionais de fornecimento de matérias-primas, serviços e/ou recursos, ou das relações construídas com a cadeia de suprimentos do negócio (Johnson, 2011).

Atualmente é comum encontrar organizações concorrentes que mantêm, por exemplo, relações de colaboração, 'subvertendo' a lógica do mercado capitalista - onde a individualidade e a busca por lucros e benefícios individuais são alguns dos principais objetivos - e realizando atividades em conjunto, consequentemente alcançando níveis de interdependência que tornam essa dinâmica ainda mais complexa e difícil de ser estudada, mensurada e compreendida.

Tais relações de colaboração podem ocorrer entre duas ou mais organizações, e neste último caso pode-se caracterizar como uma rede interorganizacional. 
No caso das redes interorganizacionais, a complexidade das relações é um dos pontos que chamam atenção. Não estão em jogo apenas relações sociais ou econômicas, mas também relações organizacionais, de forma que vários interesses devem ser considerados.

Para Inkpen e Tsang (2005), as redes proveem as empresas participantes com acesso a conhecimentos, recursos, mercados e/ou tecnologias. Inkpen e Tsang (2005) apontam a capacidade de transferência de conhecimentos como um dos elementos de grande importância e uma das maiores vantagens das redes interorganizacionais. No entanto, para que essa transferência ocorra, é necessária a criação de laços fortes entre as empresas e entre os atores envolvidos nos processos de criação e compartilhamento de conhecimento.

Em seu trabalho sobre grupos sociais, Granovetter (1973) abordou as ligações entre atores delimitando dois tipos de conexão: os laços fracos e os laços fortes. Para Granovetter (1973), a força de um laço pode ser vista como a combinação de tempo, de intensidade emocional, de intimidade e de serviços recíprocos que caracterizam um laço.

Segundo Granovetter (1973), quando os grupos sociais alcançam graus de coesão tão intensos a ponto de auxiliar na tomada de decisões mais consistentes e coerentes, como um consenso entre o grupo, eles podem ser vistos como indivíduos que criaram laços fortes. Nesse contexto, Granovetter (1973) apresentou a importância dos laços fortes entre os atores para uma maior coesão nas decisões e maiores chances de engajamento.

Por outro lado, Granovetter (1973) apontou que laços fracos entre os atores e dentro dos grupos são também necessários, pois é por meio dessas conexões que novas informações e conhecimentos podem ser compartilhados, tendo em vista que os indivíduos/atores que possuem laços fortes geralmente compartilham das mesmas crenças, experiências, e, consequentemente, informações e conhecimentos. Assim, por meio das conexões de laços fracos novas visões de mundo, novas posturas, além de diferentes informações e conhecimentos, são compartilhadas com a rede, contribuindo para a criação de novos tipos de conhecimentos e de experiências.

Corroborando os estudos de Granovetter (1983), Inkpen e Tsang (2005) salientam que os laços fracos fornecem às empresas informações valiosas. No entanto, somente a partir do momento em que se constroem laços fortes entre os indivíduos é que essas informações têm o potencial de se tornar conhecimento e serem utilizadas dentro das empresas.

Segundo Inkpen e Tsang (2005), os laços fortes podem ser criados por meio de ações de parceria repetidas com os mesmos atores, o que fortalece as relações e os laços criados. Nesse sentido, algumas das vantagens da criação e fortalecimento de laços fortes surgem do aumento da confiança entre os atores, além da reciprocidade no compartilhamento de recursos e das perspectivas de projetos conjuntos no longo prazo.

Com base nesses conceitos, utilizam-se os apontamentos de Grabher e Ibert (2006), que indicam que a criação de redes entre organizações diversas é uma característica fundamental para as empresas se manterem no mercado. Os autores dizem que o know how (saber como) está sendo substituído pelo know whom (saber quem), de forma que as redes proporcionam contatos e laços variados, podendo servir, dentre outros elementos, como base de consulta sobre determinados temas/especialidades, enfatizando a importância de manter uma rede de 
relações com pessoas de dentro da organização e de fora dela, ou seja, a busca por criar laços fortes e laços fracos com diferentes atores.

Utilizando-se da teoria dos laços de Granovetter (1973), Grabher e Ibert (2006) sugerem que as redes pessoais duram mais tempo do que as redes interorganizacionais, as quais, segundo eles, tendem a se manter ativas apenas enquanto há projetos em desenvolvimento. Já as redes pessoais tendem a manter a relação, possibilitando o surgimento e criação de laços fortes entre os atores envolvidos. Desta forma, os autores enfatizam a necessidade de se dedicar atenção às relações entre as pessoas que compõem a rede, e não apenas às relações empresariais e/ ou comerciais.

De acordo com Nohria e Eccles (1992 como citado em Balestrin \& Vargas, 2002), três razões principais foram identificadas no final do século XX e início do século XXI para o aumento do interesse no tema de redes interorganizacionais: a) a emergência de novas formas de competição, onde estão presentes relações laterais intra e interfirmas, deixando de lado posturas hierárquicas e rígidas; b) o surgimento de tecnologias da informação e comunicação (TICs), que tem aumentado as possibilidades de inter-relação entre empresas; e c) a consolidação da análise de redes como uma disciplina acadêmica.

Conforme apontam Balestrin e Vargas (2002), o campo de estudos sobre redes interorganizacionais é interdisciplinar e possibilita abordagens oriundas de diversas áreas do conhecimento.

Além disso, e conforme discutido anteriormente, algumas questões que levam organizações a se organizarem em rede são as possibilidades de compartilhamento de recursos, tanto físicos e financeiros como de conhecimentos, ou mesmo a possibilidade de compartilhamento de riscos relacionados com novos empreendimentos, dentre outros fatores.

Nesse contexto, parte-se da premissa de que os interesses que levam as organizações a formarem redes que podem vir a se tornar redes de conhecimento - podem convergir no sentido de desenvolvimento de produtos e serviços (criação de artefatos), redes de Pesquisa e Desenvolvimento (P\&D) e/ou outras formas de colaboração interorganizacional.

Para Balestrin e Verschoore (2009), "a cooperação entre organizações emerge como consequência de agentes individuais buscando satisfazer aos próprios interesses; isto é, as empresas colaboram entre si visando a ganhos que não poderiam obter de forma isolada" (p. 48). Desta forma, com as redes de conhecimento, adotandose a premissa de que são também redes colaborativas, a situação apresenta-se como semelhante à situação da formação das redes de colaboração, conforme apresentado na próxima seção.

\section{Redes de Conhecimento}

O estudo das redes de conhecimento teve início na década de 1990 (Pérez \& Rodríguez, 2005; Tomaél, 2008), quando as organizações passaram a perceber o valor do conhecimento para o desenvolvimento de seus produtos e serviços e iniciaram a busca de maneiras mais efetivas de criar conhecimentos e utilizá-los no seu dia a dia.

Johnson (2012) aponta que as redes de conhecimento são vistas como grupos de colaboração entre 
pessoas e entre empresas, baseados, fundamentalmente, no compartilhamento de conhecimentos. O autor aponta a importância das redes formais e das informais para a troca de informações e conhecimentos entre os indivíduos. São as relações entre os indivíduos que possibilitam a criação e o compartilhamento de conhecimentos dentro das empresas e entre elas, de forma que a atenção nas redes de conhecimento deve ser dada às relações, e não à estrutura em si (Johnson, 2012).

Para Tomaél (2008), a expressão 'redes de conhecimento' é utilizada para designar variados modelos de trabalhos de cooperação, como redes de gestão do conhecimento, alianças estratégicas, redes de especialistas, redes de informações, comunidades de prática, redes de conhecimento virtuais, dentre outros elementos e formas de redes de cooperação. Segundo a autora supramencionada, uma das principais motivações da estruturação desse tipo de rede é o compartilhamento de conhecimentos, em sua maioria tácitos, pois são mais difíceis de serem compartilhados e absorvidos.

Para Teixeira (2011), as redes de conhecimento podem ser vistas como espaços para a troca de informações e experiências geralmente realizada entre profissionais de diversas áreas.

Liu et al. (2013) salientam que o conceito de rede de conhecimento ainda pode levar a interpretações variadas, pois não há uma definição padrão aceita para o termo. No entanto, os autores apontam que, mesmo sem uma concordância geral, os conceitos e proposições de delimitações para as RC congregam elementos de redes de cooperação voltadas para o conhecimento.

Como o próprio nome indica, o elemento central e objeto de interesse dos membros de uma RC é o conhecimento, em suas mais variadas formas. Tanto o compartilhamento do conhecimento já possuído pelos atores da rede como a criação de novos conhecimentos são objeto de estudo e da prática das RC.

Em um contexto de redes, Huggins et al. (2008) apontam que o conhecimento não é visto apenas como um elemento de competitividade para desenvolvimento de produtos e serviços, mas também para o desenvolvimento e aprimoramento de territórios, e mesmo de regiões. Os autores ainda apontam que as universidades e outras instituições de ensino e de pesquisa podem ser vistas como fontes de conhecimentos científicos e tecnológicos capazes de auxiliar no desenvolvimento de determinadas regiões, podendo também compor redes de conhecimento e redes de cooperação com os mais variados setores de atividades. Segundo eles, a transferência de conhecimentos e tecnologias é um dos elementos centrais nesse processo.

Para Tomaél (2008), dentro das redes, tanto a criação como o compartilhamento de conhecimentos tácitos requerem a adoção de métodos de trabalho em colaboração, além do estabelecimento de relacionamentos e de confiança entre os atores. Ainda segundo Tomaél (2008):

Nas redes de conhecimento, a informação carece de interpretação. Normalmente é subjetiva e provém de um ator que coopera na rede com sua bagagem intelectual, cultural e organizacional. É essa informação, e seu compartilhamento, o foco do estudo das redes de conhecimento e é por meio dela que o conhecimento individual pode ser o mote para parcerias que tragam benefícios recíprocos. (s.p.) 
A cooperação, nesse sentido, consiste em abandonar o individualismo, saber tolerar, saber ceder. É preciso estar livre e acessível para a ampliação de fronteiras de ações individuais e organizacionais, ou para o recuo destas, e estar predisposto a compartilhar dados, informações e conhecimentos para o bem comum e para o atendimento dos objetivos da rede. Há a necessidade de criação de um senso de coletividade, onde os interesses coletivos passam a ser o centro das relações, deixando de lado posturas individualistas e unilaterais (Tomaél, 2008).

Desta forma, compreender as características e especificidades das redes de conhecimento e da possibilidade de compartilhamento e criação de conhecimentos é um ponto que merece atenção e discussões, o que é apresentado na próxima seção.

\section{Elementos fundamentais para a constituição de redes de conhecimento}

As redes de conhecimento podem ser vistas como uma forma de socialização de informações e conhecimentos entre os indivíduos e/ou organizações participantes da rede e, também, como um espaço para a criação de novos conhecimentos.

Conforme apontado por Tomaél (2008), alguns elementos são necessários para que uma rede de conhecimentos seja efetiva: a) troca de informações entre várias empresas; b) estabelecimento de um intercâmbio de ideias; c) desenvolvimento de visão estratégica; d) definição de áreas de atuação; e) análise conjunta dos problemas e solução em comum; e f) definição das contribuições dos parceiros.

Partindo desses elementos apontados por Tomaél (2008), verifica-se que para o estabelecimento de uma RC é necessária a interação efetiva entre os integrantes da rede para que a troca de informações e conhecimentos ocorra.

Como qualquer projeto, uma RC deve ser planejada e ter algumas definições básicas, como áreas de interesse e/ou de atuação, objetivos definidos, visão compartilhada pelos membros da rede e processos decisórios com a participação de todos.

Nesse sentido, vê-se a possibilidade de as RC serem criadas tanto a partir de atores individuais como de organizações, traduzindo interesses comuns e buscando, por meio de metodologias próprias, compartilhar e criar novos conhecimentos a partir da interação dos indivíduos da rede.

Johnson (2011) aponta que, com relação aos indivíduos, é preciso lhes fornecer autonomia para a busca de informações e, consequentemente, para o seu compartilhamento, bem como possibilidade de aprendizado individual e coletivo. Além disso, o autor supramencionado cita a necessidade de proporcionar um ambiente que favoreça e encoraje o aprendizado e a criação do conhecimento, pensamento este já utilizado por autores como McGee e Prusak (1998) e Nonaka e Takeuchi (1997), que identificam que uma das características de empresas e programas voltados para o compartilhamento e criação do conhecimento é o estabelecimento de um ambiente que proporcione aos indivíduos os elementos, tanto físicos e normativos como culturais e comportamentais, necessários para o compartilhamento do conhecimento.

Com base nesses conceitos, é possível perceber a inter-relação das redes de conhecimento com as metodologias de criação e compartilhamento do conhecimento estudadas pela Gestão do Conhecimento (GC). 
De acordo com Alvarenga Neto (2008), um dos objetivos da GC é criar condições dentro da organização para que ela e seus membros possam utilizar as informações e conhecimentos disponíveis, além de trabalharem na criação de novos conhecimentos.

Levando isso para um ambiente interorganizacional, acredita-se que a lógica seja a mesma: criar condições para que os indivíduos possam interagir, compartilhando informações e conhecimentos e criando novos conhecimentos com base nesse processo de interação e de relações de cooperação.

Sabendo-se que a criação e o compartilhamento de conhecimentos dentro das RC dependem de interação entre os atores, pode-se fazer uma relação com o processo de conversão do conhecimento de Nonaka e Takeuchi (1997), que indicam que para que o conhecimento seja criado e compartilhado é necessária a interação entre os indivíduos tornando possível a articulação do conhecimento tácito individual para o conhecimento explícito.

Nesse contexto, Nonaka e Takeuchi (1997) apresentam um modelo que busca a conversão do conhecimento tácito dos indivíduos para conhecimento explícito por meio do processo $\mathrm{SECl}$, que contém as etapas de Socialização, Externalização, Combinação e Internalização do conhecimento, conforme segue:

a. Socialização: compartilhamento e criação de conhecimento tácito através de experiência direta. A socialização acontece de indivíduo para indivíduo, de conhecimento tácito para conhecimento tácito;

b. Externalização: articulação de conhecimento tácito através do diálogo e da reflexão. A externalização ocorre de indivíduo para grupo, de conhecimento tácito para explícito;

c. Combinação: sistematização e aplicação do conhecimento e da informação, ocorrendo do grupo para a organização. A combinação é feita de conhecimento explícito para conhecimento explícito;

d. Internalização: aprendizado e aquisição do novo conhecimento tácito à prática organizacional. A internalização ocorre de organização para indivíduo e é feita de conhecimento explícito para conhecimento tácito.

As quatro formas de conversão do conhecimento se retroalimentam, dando continuidade ao processo. Percebe-se que por meio dos processos de conversão dos conhecimentos tácitos para conhecimentos explícitos é possível fomentar a criação de novos conhecimentos dentro das organizações por meio de processos contínuos de conversão dos conhecimentos. Isso também pode ser aplicado nos contextos de interação entre indivíduos de organizações diferentes, que é o caso das RC, onde a relação interorganizacional proporciona elementos para a criação de novos conhecimentos compartilhados entre os membros da rede.

Desta forma, entende-se que a efetividade das RC depende também da estruturação de práticas e metodologias capazes de fomentar a interação entre os participantes e operacionalizar maneiras de compartilhar conhecimentos individuais e criar novos conhecimentos com base na interação entre os indivíduos.

Conforme aponta Teixeira (2011), "o processo de desenvolvimento do conhecimento, individual ou corporativo, deve ser formal, e as redes de conhecimento devem dispor de ferramentas e procedimentos que contribuam para a formalização deste conhecimento". (p. 19) 
Assim, a relação entre as metodologias de Gestão do Conhecimento e a efetividade das Redes de Conhecimento se torna evidente e necessária: as redes tendem a proporcionar a interação necessária para que o conhecimento dos indivíduos seja compartilhado; já as metodologias de GC podem contribuir para a estruturação de práticas que consigam tornar o conhecimento gerado dentro das redes algo passível de ser articulado e, consequentemente, utilizado de forma mais efetiva.

\section{Metodologia}

Para responder aos objetivos propostos, a pesquisa iniciou-se com uma investigação bibliográfica para traçar um panorama sobre o tema em questão. Foram selecionados materiais de referência na área e autores mais citados, utilizando-se como critério de seleção o número de citações encontradas no Google Acadêmico.

Complementando essa investigação inicial, optou-se pela realização de uma pesquisa bibliométrica sobre o tema Redes de Conhecimento. Os procedimentos adotados na pesquisa bibliométrica partiram da identificação dos descritores da pesquisa (as palavras-chave a serem buscadas nas bases de dados). Foram definidos, nessa etapa, os descritores a seguir: 'redes de conhecimento'; 'redes de conhecimento' and 'gestão do conhecimento'; 'redes de conhecimento' and 'ambiente organizacional'; 'redes de conhecimento' and 'cooperação interorganizacional'; 'redes de conhecimento' and 'colaboração interorganizacional'; 'redes de conhecimento' and 'redes interorganizacionais'. Os buscadores foram utilizados em português e inglês.

Em seguida, verificou-se a aderência dos termos de pesquisa ao trabalho proposto por meio de buscas iniciais em algumas bases de dados. Após identificada a aderência, foram selecionadas as bases de dados. Em seguida, a busca foi realizada e os artigos encontrados foram selecionados e analisados com relação ao título e, posteriormente, com relação à aderência com o tema deste estudo. Com base nessa análise, foi definido o corpus da pesquisa para, em seguida, ser realizada a leitura e análise dos artigos no intuito de identificar as principais características das RC na literatura, conforme Figura 1.

Foram selecionadas duas bases de dados nacionais (Scielo e Capes) e três internacionais (Web of Science, Scopus e Science Direct) para verificar a produção científica sobre o tema. A coleta de dados ocorreu no período de julho a outubro de 2014 e foi repetida no período de março e abril de 2015, não retornando resultados diferentes. Salienta-se que as três bases de dados internacionais foram selecionadas por concentrarem trabalhos em várias áreas do conhecimento, incluindo as áreas interdisciplinares. Nesse contexto, e partindo da premissa de que o estudo de redes de conhecimento pode partir de áreas interdisciplinares do saber, optou-se pelas três bases informadas por fornecerem grandes quantidades de trabalhos oriundos de vários países. 
Figura 1

Etapas da pesquisa bibliométrica

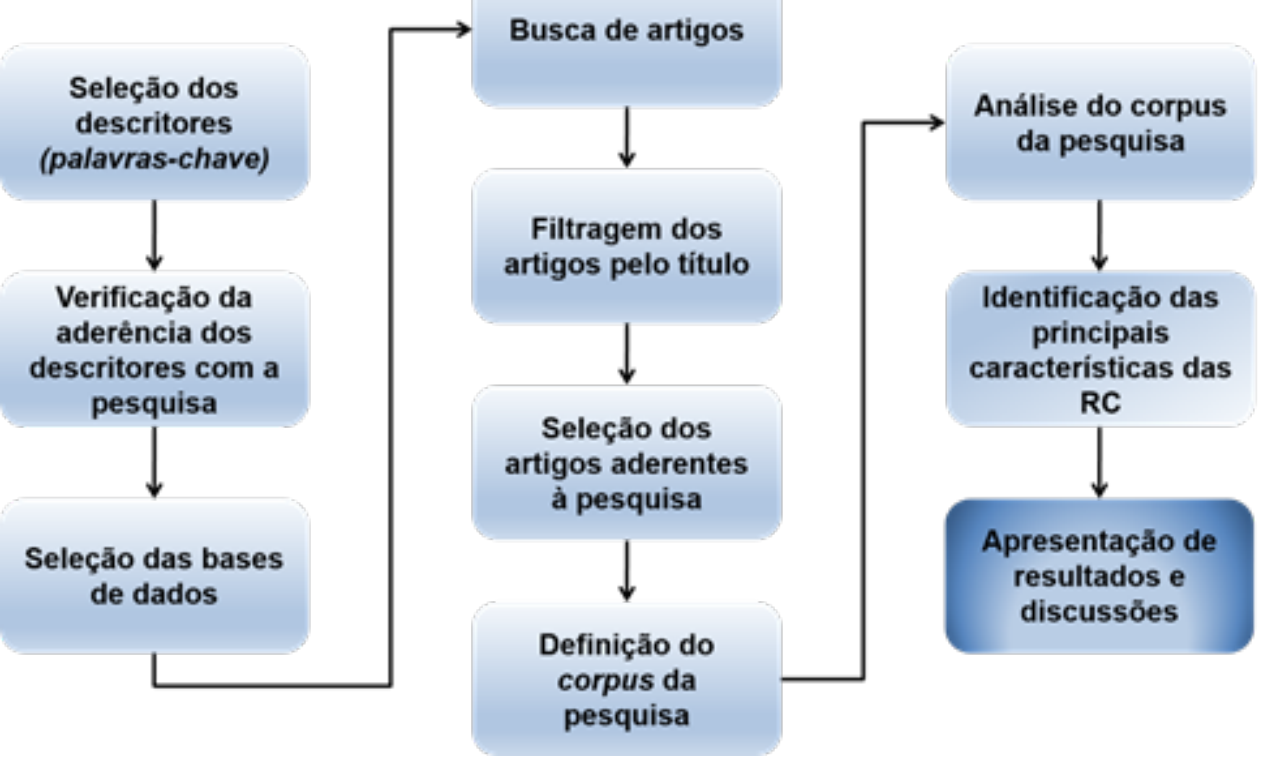

Nota. Elaboração própria (2016).

No cenário nacional foram encontrados apenas dez (10) trabalhos indexados nas bases de dados consultadas, sendo que um (1) aparecia nas duas bases, tendo-se o total de nove (9) trabalhos nacionais. No entanto, verificou-se, após leitura dos resumos, que esses trabalhos enfocam a questão das redes no contexto da produção/pesquisa acadêmica e científica, não atendendo aos objetivos deste trabalho. Já no cenário internacional a produção acadêmica apresenta quantidades superiores, indicando uma maior maturidade dos estudos sobre redes de conhecimento, no entanto ainda incipientes no que tange às redes de conhecimento interorganizacionais. Os resultados do cenário internacional, bem como os termos de pesquisa utilizados na consulta às bases de dados, são apresentados na Tabela 1.

Tabela 1

Resultados da pesquisa bibliométrica em bases internacionais

\begin{tabular}{|c|c|c|c|c|c|c|c|c|}
\hline \multirow[t]{2}{*}{ Termos consultados } & \multicolumn{2}{|c|}{ Web of Science } & \multicolumn{2}{|c|}{ Scopus } & \multicolumn{2}{|c|}{ Science Direct } & \multicolumn{2}{|c|}{ Total geral } \\
\hline & $\mathrm{n}$ & $\%$ & $\mathrm{n}$ & $\%$ & $\mathrm{n}$ & $\%$ & $\mathrm{n}$ & $\%$ \\
\hline Redes de Conhecimento & 6 & $1 \%$ & 2 & $0 \%$ & 212 & $42 \%$ & 220 & $10 \%$ \\
\hline \multicolumn{9}{|c|}{ Redes de Conhecimento e Gestão do } \\
\hline Conhecimento & 0 & $0 \%$ & 0 & $0 \%$ & 69 & $14 \%$ & 69 & $3 \%$ \\
\hline \multicolumn{9}{|c|}{ Redes de Conhecimento e Ambiente } \\
\hline Organizacional & 0 & $0 \%$ & 0 & $0 \%$ & 21 & $4 \%$ & 21 & $1 \%$ \\
\hline \multicolumn{9}{|c|}{ Redes de Conhecimento e Cooperação } \\
\hline Interorganizacional & 0 & $0 \%$ & 0 & $0 \%$ & 0 & $0 \%$ & 0 & $0 \%$ \\
\hline \multicolumn{9}{|c|}{ Redes de Conhecimento e Colaboração } \\
\hline Interorganizacional & 0 & $0 \%$ & 0 & $0 \%$ & 0 & $0 \%$ & 0 & $0 \%$ \\
\hline
\end{tabular}




\begin{tabular}{|c|c|c|c|c|c|c|c|c|}
\hline \multirow[t]{2}{*}{ Termos consultados } & \multicolumn{2}{|c|}{ Web of Science } & \multicolumn{2}{|c|}{ Scopus } & \multicolumn{2}{|c|}{ Science Direct } & \multicolumn{2}{|c|}{ Total geral } \\
\hline & $\mathrm{n}$ & $\%$ & $\mathrm{n}$ & $\%$ & $\mathrm{n}$ & $\%$ & $\mathrm{n}$ & $\%$ \\
\hline \multicolumn{9}{|l|}{ Criação de Conhecimento e Redes } \\
\hline Interorganizacionais & 1 & $0 \%$ & 0 & $0 \%$ & 1 & $0 \%$ & 2 & $0 \%$ \\
\hline Knowledge networks & 627 & $88 \%$ & 800 & $83 \%$ & 196 & $39 \%$ & 1623 & $75 \%$ \\
\hline \multicolumn{9}{|l|}{ Knowledge networks and Knowledge } \\
\hline Management & 78 & $11 \%$ & 129 & $13 \%$ & 1 & $0 \%$ & 208 & $10 \%$ \\
\hline \multicolumn{9}{|l|}{ Knowledge networks and Organizational } \\
\hline Environment & 1 & $0 \%$ & 1 & $0 \%$ & 1 & $0 \%$ & 3 & $0 \%$ \\
\hline \multicolumn{9}{|l|}{ Knowledge networks and } \\
\hline Interorganizational Cooperation & 0 & $0 \%$ & 2 & $0 \%$ & 1 & $0 \%$ & 3 & $0 \%$ \\
\hline \multicolumn{9}{|l|}{ Knowledge networks and } \\
\hline Interorganizational Colaboration & 0 & $0 \%$ & 0 & $0 \%$ & 0 & $0 \%$ & 0 & $0 \%$ \\
\hline \multicolumn{9}{|l|}{ Knowledge creation and } \\
\hline Interorganizarional Networks & 0 & $0 \%$ & 26 & $3 \%$ & 0 & $0 \%$ & 26 & $1 \%$ \\
\hline Total & 713 & $100 \%$ & 960 & $100 \%$ & 502 & $100 \%$ & 2175 & $100 \%$ \\
\hline
\end{tabular}

Nota. Dados da pesquisa (2016)

Deste total de 2.175 trabalhos retornados, os artigos foram analisados de acordo com o título, buscando encontrar similaridades com o interesse desta pesquisa. Após essa primeira análise, chegou-se ao número de 168 artigos alinhados, pelo título, com o tema aqui proposto. No entanto, os artigos ainda foram analisados para buscar duplicidades, o que eliminou 17 registros duplicados, alcançando-se o total de 151 trabalhos. Após atingir esse número (151), os artigos foram analisados novamente com relação ao tema abordado e seu alinhamento com o tema central deste estudo (redes de conhecimento). Nessa análise obteve-se o número de 95 artigos publicados no cenário internacional alinhados pelo título com o tema central desta pesquisa.

Desses 95 artigos encontrados nas bases de dados internacionais consultadas, após a leitura dos resumos e das palavras-chave, obteve-se o resultado de 32 trabalhos alinhados com o tema de pesquisa e disponíveis na internet, indicando uma pesquisa ainda incipiente no que tange aos estudos em redes de conhecimento.

A partir desses resultados, seguiu-se a leitura dos trabalhos e a elaboração da pesquisa bibliográfica buscando identificar as principais abordagens sobre as redes de conhecimento, bem como características das RC.

\section{Contribuições teóricas sobre as Redes de Conhecimento}

Conforme indicado na pesquisa bibliométrica, os trabalhos encontrados no cenário nacional (09) indicam uma produção acadêmica incipiente no que tange às redes de conhecimento interorganizacionais. Verificou-se que esses trabalhos enfocam estudos voltados para as redes de conhecimento de pesquisadores e de produção científica em várias áreas do saber. Outro ponto observado é que existem trabalhos sobre o tema em outras bases de dados, não indexados nas bases consultadas, o que justifica a não agregação desses trabalhos no corpus teórico desta pesquisa. 
Por outro lado, no cenário internacional, embora a maioria dos trabalhos encontrados nas bases de dados consultadas esteja relacionada com as redes de conhecimento de pesquisadores e da produção científica, foi possível perceber que os trabalhos voltados para as redes de conhecimento interorganizacionais estão recebendo mais atenção e crescendo em número de publicações. Com relação à amostra utilizada, o número de 32 trabalhos selecionados corresponde a trabalhos alinhados com a proposta deste estudo (redes de conhecimento interorganizacionais). Os demais resultados retornados da pesquisa bibliométrica indicaram grande incidência de trabalhos sobre as RC na produção de conhecimento científico, o que não era foco desta pesquisa.

Em termos gerais, observou-se que os trabalhos alinhados com os interesses de pesquisa deste estudo abordam, entre outros temas, os elementos necessários para a formação das RC, indicando aspectos como:

- Alinhamento de objetivos dos atores envolvidos;

- Compartilhamento de recursos estruturais, físicos e financeiros;

- Troca de informações e conhecimentos entre as organizações e indivíduos participantes;

- Proximidade geográfica;

- Relações de cooperação a partir de iniciativas conjuntas.

Conforme foi possível perceber em alguns trabalhos consultados (Maggioni \& Uberti, 2009; Schönström, 2005; Aular \& Pereira, 2009), a temática do desenvolvimento regional por meio das redes de conhecimento surgiu como um ponto favorável para a criação e manutenção das RC. Autores como Maggioni e Uberti (2009) e Huggins et al. (2008) apontam que a proximidade geográfica das organizações participantes de uma rede pode contribuir para que outras empresas se desenvolvam, na medida em que os núcleos industriais e comerciais necessitam de serviços, matéria-prima e outros recursos que podem ser fornecidos por outras organizações. Com isso, o desenvolvimento de determinadas localidades pode ser impulsionado pelas ações de colaboração entre as empresas de uma RC.

No entanto, a proximidade geográfica é um fator que pode ser contornado, principalmente, com a utilização de ferramentas de tecnologias da informação e comunicação, que auxiliam no compartilhamento de informações e conhecimentos, além de facilitarem e agilizarem o processo comunicacional entre empresas, um dos elementos fundamentais para a estruturação das redes. Nesse contexto, Maggioni e Uberti (2009) salientam que a distância geográfica se torna um empecilho maior quando as empresas não possuem a mesma finalidade, ou seja, quando as empresas atuam em áreas/setores diferentes. Os autores afirmam que isso não inviabiliza o compartilhamento de dados, informações e conhecimentos, porém pode tornar esse processo menos efetivo em função das divergências de comportamento, atuação e visão de mundo.

Por outro lado, autores como Larner (2015) e Scnönström (2005) indicam que as RC estão investindo na colaboração com universidades e centros de pesquisa, que lhes fornecem conhecimentos e tecnologias atualizados e, em geral, comprovados, o que tem o potencial de maximizar os resultados da rede e de seus participantes além de aprimorar os processos de inovação dentro das empresas. 
Rajan e Rajan (2013) apontam que as universidades podem ser boas opções para a criação e disseminação do conhecimento. No entanto, não apresentam grandes novidades com relação à adoção de novas práticas relacionadas com o ato de compartilhar o conhecimento. Para Rajan e Rajan (2013), as RC são oportunidades para as universidades compartilharem seus conhecimentos, além de criar novos conhecimentos por meio da interação com outros atores.

Aular e Pereira (2009) apresentam as universidades como elos das RC, capazes de dar respostas às demandas de inovação e de informação das organizações. Por outro lado, de acordo com as autoras, as universidades em si mesmas constituem-se como redes de conhecimento na medida em que congregam profissionais diversos e atuam na criação e difusão do conhecimento em suas mais variadas formas. Aular e Pereira (2009) ainda apontam que para se criar e compartilhar conhecimento é necessário o estabelecimento de redes, sejam elas internas à organização, sejam externas a ela.

Scnönström (2005, tradução nossa), corroborando esses apontamentos, indica que:

As redes dão suporte à inovação porque os indivíduos se tornam conscientes de novas tecnologias e novos conhecimentos por meio de atividades que ampliam suas fronteiras, como o networking. Nessas atividades os indivíduos têm acesso a conhecimentos que podem ser relevantes para suas próprias organizações. (p.

19)

Mentzas et al. (2006) afirmam que, quando duas ou mais organizações pretendem compartilhar informações e conhecimentos, um dos primeiros aspectos a serem observados são as fontes de informações e conhecimentos dentro da empresa. Mentzas et al. (2006) indicam que esses conhecimentos podem estar dispostos em processos, produtos, rotinas, ferramentas e mesmo com as próprias pessoas. Nesse sentido, os conhecimentos que pertencem aos indivíduos (tácitos) devem ser articulados e trabalhados de maneira que possam ser compartilhados.

Llerena e Ozman (2013) indicam que os conhecimentos tácitos dos indivíduos necessitam de codificação para que possam ser trabalhados e compartilhados com mais facilidade. No entanto, quando em redes, as organizações - e os indivíduos que fazem parte dela - nem sempre possuem o mesmo nível de informações, conhecimentos e experiências, o que às vezes se torna um empecilho para o compartilhamento em função de que algumas organizações podem contribuir menos com a rede do que outras (Llerena \& Ozman, 2013).

Em contrapartida, como a criação e manutenção das RC dependem da capacidade das organizações de compartilhar dados, informações e, sobretudo, conhecimentos, um dos maiores desafios é realizar a articulação do conhecimento tácito dos indivíduos e transformá-lo em conhecimento explícito (ou conhecimento codificado), passível de compartilhamento e combinação com outros conhecimentos (Schönström, 2005; Mentzas et al., 2006), conforme visto a partir do processo de conversão do conhecimento de Nonaka e Takeuchi (1997).

Para isso, as organizações contam com as ferramentas de TIC, além dos sistemas de informações, que lhes possibilitam atuar de forma mais efetiva na coleta, processamento, armazenamento e disseminação de informações e conhecimentos. 
Aular e Pereira (2009) apontam que as ferramentas de TIC têm grande influência no sucesso das ações de compartilhamento do conhecimento e mesmo na criação de novos conhecimentos por meio da interação entre os atores de uma rede.

Ainda, é possível observar a constituição de redes de conhecimento virtuais, utilizando-se de plataformas on-line e de ferramentas de TIC para o compartilhamento de informações e conhecimentos e para a criação de novos conhecimentos de forma coletiva, o que se torna uma alternativa com relação à distância geográfica de indivíduos e organizações.

Nesse contexto, a Tabela 2 apresenta uma síntese dos principais elementos percebidos como comuns às redes de conhecimento. Por meio da literatura consultada, verificou-se que elementos como comunicação entre os atores da RC, presença constante de utilização de ferramentas de TIC, normas e procedimentos-padrão adotados na rede, bem como a criação de uma cultura voltada para o compartilhamento de informações e conhecimentos, são alguns dos aspectos gerais das redes de conhecimento.

\section{Tabela 2}

Redes de Conhecimento e suas características

\section{Aspectos gerais}

- Ambiente de cooperação entre empresas e indivíduos;

- Cultura voltada para o compartilhamento de informações e conhecimentos

- Presença de ferramentas de TIC nos processos de compartilhamento de informações e conhecimentos

- Comunicação livre entre os atores da rede

- Estabelecimento de normas e procedimentos básicos

\section{Elementos fundamentais}

- Criação de identidade entre os membros da rede

- Estabelecimento de regras claras para a participação na rede

- Interação entre os participantes

- Compartilhamento de dados, informações e conhecimentos

- Objetivos comuns

- Planejamento e definição de ações estratégicas

- Fortalecimento dos laços entre os atores

\section{Possíveis resultados}

- Compartilhamento de recursos físicos, estruturais e financeiros

- Acesso a dados e informações privilegiados

- Criação de conhecimentos diversos

- Fortalecimento da rede em termos de vantagens competitivas

- Aprimoramento técnico e profissional para os indivíduos

- Desenvolvimento tecnológico e organizacional para as organizações participantes

Nota. Elaborado pelos autores com base na literatura consultada (2016)

Verificou-se ainda que elementos como planejamento e definição de estratégias de atuação da rede, definição de regras para o ingresso dos participantes, o encontro de objetivos comuns para os membros do grupo, bem como a criação de identidade entre os integrantes da rede, são alguns dos elementos fundamentais para a constituição de uma RC efetiva.

Já com relação aos benefícios da criação e manutenção de uma RC, verificou-se que tanto os atores individuais como as organizações participantes da rede podem obter vantagens a partir da cooperação e da efetividade da rede. Fatores como o aprimoramento técnico e profissional dos indivíduos, criação de laços fortes 
com outros atores, desenvolvimento tecnológico e organizacional para as empresas participantes, além de criação de novos conhecimentos, compartilhamento de recursos e acesso privilegiado a informações e conhecimentos, são aspectos que justificam e recompensam a participação efetiva nas RC.

Schönström (2005) ainda apresenta a melhoria na criação do conhecimento, tanto interna como interorganizacionalmente, e o aprimoramento no aprendizado dos trabalhadores como fatores positivos da estruturação das RC. Por outro lado, o autor indica que quando as redes são muito grandes o excesso de atores reduz a efetividade dos processos comunicacionais e, consequentemente, do compartilhamento de informações e conhecimentos.

Para Pérez e Rodríguez (2005), as redes de conhecimento constituem a máxima expressão do homem como um produtor de conhecimentos. Para as autoras supracitadas, os indivíduos têm a necessidade de trocar, compartilhar e transferir o que aprendem e o [conhecimento] que criam a partir da interação com outros indivíduos. Na rede isso se torna possível, além de proporcionar aos participantes a criação e a aquisição de novos conhecimentos. Pérez e Rodríguez (2005) ainda salientam que as redes tendem a se conectar com outras redes, algumas vezes formando comunidades, geralmente compostas por empresas, profissionais e/ou especialistas em determinados assuntos.

\section{Considerações Finais}

Atendendo aos objetivos propostos, este trabalho buscou investigar, com base em uma pesquisa bibliográfica, as principais características das redes de conhecimento, bem como as possibilidades de criação do conhecimento dentro das redes.

Verificou-se que as redes de conhecimento são espaços de colaboração entre atores diversos, que buscam compartilhar e criar novos conhecimentos. Para a existência e manutenção das RC é preciso que haja a criação de uma identidade comum entre os seus participantes, além de definições básicas de regras para ingresso e participação na rede. Outros fatores como cultura voltada para o compartilhamento de informações e conhecimentos, além de presença de ferramentas de TIC para facilitar a comunicação e o compartilhamento de dados e informações, são necessários para que se estabeleça uma rede de conhecimentos.

Observou-se ainda, nos trabalhos consultados, que embora as redes surjam de maneira informal, em muitos casos, a estruturação de metodologias e definições de estratégias para a atuação da RC se faz necessária.

Com relação à efetividade das RC, identificou-se a interação entre os membros da rede e o compartilhamento de informações e conhecimentos como elementos fundamentais para que novos conhecimentos possam ser gerados e para que todos os membros da rede se beneficiem de sua atuação. Nesse sentido, percebeu-se a inter-relação entre a teoria das RC e as metodologias de Gestão do Conhecimento (GC). Por meio de metodologias e práticas de GC, é possível articular o conhecimento dos indivíduos de forma a torná-lo passível de compartilhamento entre os atores que compõem a rede.

Desta forma, tornou-se possível identificar elementos e características comuns às RC, além de identificar alguns benefícios e possíveis resultados da atuação conjunta. Conforme apresentado nas seções anteriores, 
a atuação em rede e a cooperação voltada para o compartilhamento e criação do conhecimento podem oferecer aos membros da rede o acesso a dados, informações e conhecimentos privilegiados, o aprimoramento técnico e profissional dos indivíduos, a aquisição de vantagens competitivas e o desenvolvimento tecnológico e organizacional para as organizações participantes da rede.

Nesse contexto, verifica-se que o estudo de redes de conhecimento interorganizacionais ainda é incipiente, carecendo de mais estudos práticos e discussões acerca de metodologias para sua estruturação.

No entanto, a pesquisa teórica não supre as demandas de conhecimento científico sobre as RC, o que se torna uma das limitações deste estudo. Outro ponto que necessita de mais atenção diz respeito à investigação empírica da possibilidade de utilizar metodologias de gestão do conhecimento na estruturação e manutenção das RC. Nesse sentido, sugere-se que sejam realizadas novas pesquisas teóricas e empíricas sobre as redes de conhecimento interorganizacionais buscando aumentar o número de trabalhos acadêmicos, principalmente no cenário nacional, e encontrar novas evidências sobre as RC. 


\section{Referências}

Alvarenga Neto, R. C. D. (2008). Gestão do Conhecimento em Organizações: proposta de mapeamento conceitual integrativo. Saraiva.

Aular, Y. M., \& Pereira, R. T. (2009). Aproximación para el diseño de una red de conocimento intra y extra universitaria. Revista de Ciencias Sociales, 15(2), 284-295.

Balestrin, A., \& Vargas, L. M. (2002). Evidencias teóricas para a compreensão das redes interorganizacionais. Anais do 2o Encontro de Estudos Organizacionais, Recife.

Balestrin, A., \& Verschoore, J. (2009). Redes de cooperação empresarial: estratégias de gestão na nova economia. Bookman.

DeBresson, C., \& Amesse, F. (1991). Networks of innovators: a review and introduction to the issue. Research Policy, 20, 363-379.

Grabher, G., \& Ibert, O. (2006). Bad Company? The ambiguity of personal knowledge networks. Journal of Economic Geography, 6, 251-271.

Granovetter, M. (1973). The strength of weak ties. American Journal of Sociology, 78(6), 1360-1380.

Huggins, R., Johnston, A., \& Steffenson, R. (2008). Universities, knowledge networks and regional policy. Cambridge Journal of Regions, Economy and Society, 1, 321-340.

Inkpen, A. C., \& Tsang, E. W. K. (2005). Social capital, networks and knowledge transfer. Academy of Management Review, 30(1), 146-165.

Johnson, J. D. (2011). Gestão de Redes de Conhecimento. Editora Senac SP.

Johnson, J. D. (2012). Knowledge Networks: dilemmas and paradoxes. International Journal of Information Management, 32, 347-353.

Larner, W. (2015). Globalising knowledge networks: universities, diaspora strategies, and academic intermediaries. Geoforum, 59, 197-205.

Liu, X., Jiang, T., \& Ma, F. (2013). Collective dynamics in knowledge networks: emerging trends analysis. Jounal of Infometrics, 7, 425-438.

Llerena, P., \& Ozman, M. (2013). Networks, irreversibility and knowledge creation. Journal of Evolutionary Economics, 23, 431-453.

Maggioni, M., \& Uberti, T. E. (2009). Knowledge networks across Europe: wich distance matters? Ann Reg Sci, 43, 691-720.

McGee, J., \& Prusak, L. (1998). Gerenciamento Estratégico da Informação: aumente a competitividade e a eficiência de sua empresa utilizando a informação como uma ferramenta estratégica. Campus.

Mentzas, G., Apostolou, D., Kafentzis, K., \& Georgolios, P. (2006). Inter-organizational networks for knowledge sharing and trading. Info Technol Manage, 7, 259-276.

Nonaka, I., \& Takeuchi, H. (1997). Criação do Conhecimento na Empresa. Elsevier. 
Pérez, M. C., \& Rodríguez, Y. P. (2005). Aspectos teórico-conceptuales sobre las redes y las comunidades virtuales de conocimento. Acimed, 16(6). http://bvs.sld.cu/revistas/aci/vol13_6_05/aci02605.htm.

Polanyi, M. (1958). Personal knowledge: towards a post-critical philosophy. The University of Chicago Press.

Rajan, M., \& Rajan, R. (2013). Beyond open resource access: An integrative approach to optimal knowledge networking. Knowledge Management: An International Journal, 12(1), 9-15.

Schönström, M. (2005). Creating knowledge networks: lessons from practice. Journal of Knowledge Management, 9(6), 17-29.

Teixeira, M. R. F. (2011). Redes de conhecimento em ciências e o compartilhamento do conhecimento [Tese de doutorado, Universidade Federal do Rio Grande do Sul].

Tomaél, M. I. (2008). Redes de Conhecimento. DataGramaZero (Revista de Ciência da Informação), 9(2). 